\title{
Les systèmes éducatifs européens au cœur de tensions contradictoires
}

Arlette Delhaxhe

\section{(2) OpenEdition}

1 Journals

Édition électronique

URL : http://journals.openedition.org/ries/3183

DOI : 10.4000/ries.3183

ISSN : 2261-4265

Éditeur

Centre international d'études pédagogiques

Édition imprimée

Date de publication : 1 septembre 1997

Pagination : 127-134

ISSN : 1254-4590

\section{Référence électronique}

Arlette Delhaxhe, "Les systèmes éducatifs européens au cœur de tensions contradictoires », Revue internationale d'éducation de Sèvres [En ligne], 15 | 1997, mis en ligne le 04 juillet 2013, consulté le 19 avril 2019. URL : http://journals.openedition.org/ries/3183; DOI : 10.4000/ries.3183

Ce document a été généré automatiquement le 19 avril 2019

(c) Tous droits réservés 


\title{
Les systèmes éducatifs européens au cour de tensions contradictoires
}

\author{
Arlette Delhaxhe
}

1 En cette fin de siècle, des voix de plus en plus nombreuses s'élèvent pour rappeler l'importance de l'éducation et de la formation ${ }^{1}$ et souligner l'urgence de leur accorder à nouveau la priorité. Elles émanent autant des responsables politiques que d'organismes internationaux comme la Commission européenne ${ }^{2}$, le Conseil de l'Europe ou l'Unesco ${ }^{3}$. Tous reconnaissent le rôle fondamental que doit jouer l'éducation pour aborder le tournant du troisième millénaire. Certes, chaque plaidoyer met l'accent sur des aspects différents mais on y retrouve toujours une même idée maîtresse: il s'agit de réussir l'intégration et la participation de tous dans la société multiculturelle, cognitive et mondiale ainsi que d'assurer la formation tout au long de la vie dans un monde en mutation permanente.

2 Ce discours se fait de plus en plus fort alors que, parallèlement, des mesures de restrictions budgétaires sont prises à l'encontre du système éducatif dans la majorité des pays européens. Par ailleurs, les critiques sur l'inertie et l'incapacité d'adaptation des systèmes éducatifs sont monnaie courante. Sans conteste, l'école est l'objet de tensions contradictoires : à la fois reconnue comme le moyen principal pour progresser, elle est aussi vivement critiquée pour sa lenteur à intégrer les nouvelles connaissances et technologies ou encore pour son incapacité à donner à tous les qualifications nécessaires à l'intégration dans la vie économique et sociale.

3 L'approche de l'an 2000 serait donc à la fois l'heure du bilan et des perspectives. Dans ce contexte, il n'est pas vain de rappeler combien l'éducation de base pour tous est une réalité jeune. Les premières législations en matière d'obligation scolaire datent généralement de la fin du XIX siècle. A l'époque, une grande partie de la société européenne était analphabète. Avec la massification de la scolarisation dans l'Union européenne, elle représente aujourd'hui approximativement $10 \%$. Certains se lamentent de ce taux actuel. Ils y voient la preuve de l'échec du système scolaire et de sa nécessaire transformation. D'autres l'utilisent pour souligner le progrès considérable du niveau 
d'éducation de la population opérée en moins d'un siècle. En définitive, les uns et les autres ont raison : si le progrès social et humain engendré par la scolarisation pour tous est indéniable, il est inacceptable que la société européenne de demain compte encore des citoyens qui ne tirent pas le bénéfice indispensable de leurs années de scolarisation. Ainsi, la lutte contre l'échec scolaire est devenue une priorité dans l'Union. Parallèlement, des mesures sont prises un peu partout pour tenter d'aider les jeunes sortis du système scolaire sans qualification en leur donnant une formation complémentaire.

4 Au début du siècle, les objectifs assignés à l'école et les contenus d'enseignement étaient clairement définis. Les limites entre l'éducation de base et les formations réservées à l'élite étaient nettement dessinées. Aujourd'hui, les délimitations entre les niveaux d'enseignement et les qualifications sont plus floues. Partout, l'enseignement de base poursuit toujours les mêmes finalités, à savoir doter tous les citoyens des connaissances, capacités et aptitudes pour s'intégrer dans la communauté où ils vivent ${ }^{4}$. Cependant, vu les nouveaux modes de communication et les technologies de plus en plus sophistiquées qui envahissent la vie quotidienne, l'école de base se voit dans l'obligation de redéfinir ses contenus et ses méthodes pour atteindre ses objectifs. L'enjeu est de taille et les politiques éducatives sont de plus en plus nombreuses à se fixer la tâche complexe de définir les compétences de base à acquérir par tous au terme de la scolarité obligatoire. Ce faisant, elles cherchent à conjuguer cet objectif avec la perspective de l'apprentissage tout au long de la vie. Motiver les élèves à continuer à apprendre constitue ainsi une autre finalité inscrite explicitement dans les programmes et lignes directrices de plusieurs pays.

5 C'est un fait, les exigences de qualification de plus en plus élevée ont conduit un nombre de plus en plus grand de jeunes à poursuivre leurs études bien au-delà de l'obligation scolaire. Le phénomène a débuté vers les années soixante-dix ${ }^{5}$ mais ses répercussions budgétaires, organisationnelles et sociales se font ressentir aujourd'hui avec acuité et ce, plus particulièrement au niveau de l'enseignement supérieur.

6 La définition des compétences minimales à acquérir au terme de la scolarité de base et la gestion de la massification de l'enseignement supérieur constituent donc deux nœuds importants parmi les enjeux auxquels les systèmes éducatifs européens doivent faire face. De surcroît, ces adaptations fondamentales sont à réaliser dans un contexte généralisé de décentralisation progressive de la gestion financière et administrative vers les responsables locaux et les établissements. Cependant, l'autonomie croissante accordée aux établissements s'accompagne de mesures répondant explicitement au souci de contrôler et d'évaluer la qualité de l'éducation dans son ensemble. Les politiques éducatives cherchent donc à conjuguer la responsabilisation et l'autonomie des acteurs locaux avec l'assurance de la qualité du fonctionnement et des performances de l'ensemble du système.

$7 \quad$ La recherche de ces équilibres marquera sans nul doute les débats et les mesures prises dans les différents pays de l'Union en cette fin de siècle. Quelques grandes lignes des orientations actuelles prises dans l'Union dans ces domaines/matières sont présentées cidessous. 


\section{De l'égalité d'accès aux compétences de base pour tous}

8 Partout en Europe, l'enseignement secondaire fait désormais partie du pilier de la formation de base du système éducatif: il rassemble tous les adolescents dans le prolongement normal du niveau primaire. Les certificats de fin de primaire et les examens d'entrée ont été abolis dans quasi tous les pays européens. Les pays nordiques ont fait figure de pionniers avec la mise en place d'une structure unique tout au long de la scolarité obligatoire. Aujourd'hui, même les pays à orientation plus sélective (Pays-Bas, Allemagne, Luxembourg, Autriche, Royaume-Uni) assouplissent leurs conditions d'accès et reculent progressivement le moment de l'orientation ${ }^{6}$. L'aspiration de conduire un maximum de jeunes au terme du secondaire supérieur se généralise et se concrétise. Elle impose aussi de mieux prendre en compte l'hétérogénéité du public que constituent les élèves de ce niveau.

9 On ne doit pas s'attendre dans les prochaines années à des réformes structurelles aussi importantes que celles mises en œuvre dans les décennies précédentes. Il est très probable que la majorité des pays vont gérer les changements nécessaires en maintenant leur structure actuelle. Le début $d u X X I^{e}$ siècle se caractérisera probablement par la disparition progressive des filières séparées entre formation générale, technique et professionnelle au sein même du secondaire supérieur et par l'élargissement de formations postsecondaires accessibles au plus grand nombre. Le processus est en cours. Il est largement amorcé en Suède depuis la réforme de 1995 qui a supprimé la séparation des filières professionnelles et générales dans le secondaire supérieur. Ce mouvement entraîne la nécessité de reconstruire des programmes adaptés permettant flexibilité et diversité des choix mais garantissant en même temps un "socle de compétences" identiques pour tous avant l'orientation vers une qualification professionnelle spécialisée. Sous des couvertures terminologiques différentes, la question des compétences clés n'est pas neuve mais elle prend aujourd'hui une importance accrue. Comme le souligne W. Hutmacher ${ }^{7}$, le concept, très largement utilisé, n'en est pas moins complexe et ambigu. Il recouvre à la fois les «savoirs » et les "savoir-faire » dont les jeunes citoyens ont besoin. Actuellement, les essais de définition des compétences clés dans les curricula nationaux sont nombreux. Ils sont aux prises entre plusieurs tensions : cerner les connaissances indispensables à faire acquérir, en évitant l'encyclopédisme, et tout en assurant l'apprentissage de la capacité d'apprendre.

10 Sur ce chantier de préoccupations, on trouve le souci d'intégrer les nouvelles technologies et le traitement de l'information dans les matières obligatoires du programme ainsi que celui d'améliorer l'apprentissage des langues. Ce dernier point est crucial pour la construction d'une identité européenne mais aussi dans la perspective de la mondialisation des échanges culturels et économiques. Des réformes récentes ont rendu l'apprentissage des langues de plus en plus précoce dans plusieurs pays dont la Suède, le Danemark et la France. Au vu des projets pilotes expérimentaux en cours dans de nombreux pays, on peut raisonnablement penser qu'au début du XXI ${ }^{\mathrm{e}}$ siècle, cet enseignement sera généralisé et rendu obligatoire dans toutes les écoles d'Europe dès les premières années de la scolarité obligatoire. Par contre, l'utilisation des ordinateurs dans les écoles est loin d'être généralisée. L'Islande fait figure de proue en la matière puisque près de $90 \%$ de ses écoles de base en sont équipées. L'intégration complète de ce nouveau 
moyen de communication et d'acquisition des connaissances est aussi urgent qu'incontournable. Il va supposer une restructuration radicale du matériel didactique, des méthodes d'enseignement et donc une formation appropriée des enseignants. À nouveau, l'enjeu est reconnu à tous les niveaux mais les moyens pour y faire face ne sont pas à la mesure des besoins.

11 La question des valeurs démocratiques et de la dimension européenne n'est pas absente des changements en cours dans les objectifs formulés. Tous les pays de l'Union l'ont intégrée dans la formation des enseignants. Ils s'efforcent de l'implanter progressivement dans les curricula en évitant l'atomisation disciplinaire et en introduisant ce qu'on appelle dans bon nombre de pays « les questions transversales » et les matières civiques. Ces questions font l'objet de débats récurrents en relation notamment avec la montée de la violence dans les écoles.

\section{Autonomie croissante des établissements, mais contrôle accru de la qualité et de l'efficacité}

Décentralisation et autonomie sont devenus les maîtres mots de la gestion du système éducatif. Partout, on tente de les justifier et les articuler avec l'idée de démocratisation et de participation. Il est certainement trop tôt pour mesurer jusqu'où iront les processus d'autonomie accordée aux institutions scolaires et les effets que produiront à terme les mesures prises dans ce sens. Mais c'est un fait incontestable, la majorité des pays membres de l'Union européenne ont doté ces dernières années les écoles à tous les niveaux éducatifs et/ou les autorités locales de pouvoirs de décisions inimaginables il y a vingt ans encore dans les pays réputés pour leur pouvoir centralisé. C'est du moins ce qu'illustre, par exemple, la réforme adoptée en Suède en 1995, l'autonomie scolaire introduite en Autriche en 1993 par un amendement à la loi sur l'organisation de l'école, la réforme de la LOGSE en 1990 en Espagne, etc. D’une manière générale, avec des degrés différents, il en résulte que la communauté scolaire de chaque établissement organise ou donne son avis sur toute une série de questions telles que les horaires, le budget, les programmes, la gestion du personnel.

Bien sûr, la situation n'est pas homogène entre les pays et la marge de manœuvre dont disposent les établissements aujourd'hui dans les différents pays dépend fortement de la situation avant les réformes. Ainsi, en Grèce et au Luxembourg, peu de réformes ont marqué l'administration dans ce sens ${ }^{8}$. Les pays offrant un pouvoir de décision dans des domaines aussi importants que le recrutement et la gestion du personnel et l'utilisation du budget ont également mis en place des instances spécialisées. Le rôle et les missions des chefs d'établissement se sont aussi radicalement transformés. Dans la majorité des pays, des conseils assurant la participation non seulement des enseignants mais aussi de la communauté locale ont été mis en place, mais l'ampleur de leur responsabilité varie d'un pays à l'autre. Leurs missions peuvent varier, de fonctions purement consultatives jusqu'à des pouvoirs exécutifs. Par exemple, le skolebes-tyrelse au Danemark et les Governing bodies en Angleterre disposent de compétences décisionnelles importantes.

Parallèlement, au-delà des implications locales diverses et pour s'assurer du bon fonctionnement global, on observe une préoccupation évidente des politiques éducatives à l'égard de l'évaluation du système éducatif. La création d'organismes ou de départements chargés de l'évaluation de l'efficacité et de la qualité de l'enseignement en 
sont un témoignage dans plusieurs pays. Il y a dix ans, la mise en place de la DEP (direction de l'Évaluation et de la Prospective en France, chargée entre autres d'organiser le pilotage du système éducatif) faisait figure d'exception. Aujourd'hui, non seulement le Royaume-Uni a mis en œuvre également son évaluation du National Curriculum, mais l'Espagne a créé son Instituto Nacional de Calidad y Evaluación et la Communauté française de Belgique vient de mettre en place une cellule d'observation et de pilotage; au Luxembourg, le service de coordination de la recherche et de l'innovation a été mis sur pied en 1993. Comme l'indiquent généralement les termes mêmes choisis pour les désigner, tous ces organismes sont chargés de définir des indicateurs pour mesurer la qualité de l'enseignement et, partant, de guider les politiques pour en améliorer le fonctionnement. La tâche est complexe et il n'existe pas de consensus sur le meilleur moyen de réaliser cette évaluation globale. Si les résultats et les performances font toujours parties intégrantes des préoccupations, la tendance à porter également son attention sur les processus s'accentue de plus en plus.

Il convient de l'admettre, le souci de rationaliser et de contrôler la rentabilité des investissements financiers octroyés à l'éducation participe étroitement de la volonté généreuse - exprimée pour justifier ces évaluations - d'assurer la qualité de l'enseignement pour tous.

\section{L'enseignement supérieur au pied du mur}

Une vague de réformes déferle sur l'enseignement supérieur et l'attention des politiques semble particulièrement portée sur ce niveau d'enseignement. Il est vrai que dans beaucoup de pays européens, il subit de plein fouet les effets de l'accroissement important du nombre d'étudiants. Suite à la démocratisation du niveau secondaire qui s'est opérée au cours des années soixante-dix, il était inéluctable que le phénomène se répercute sur l'enseignement supérieur dans les années quatre-vingt et, pourtant, force est de constater que les systèmes éducatifs y étaient mal préparés.

17 Tout d'abord, la longue période de récession économique subie par la majorité des pays influence radicalement les mesures prises dans l'enseignement supérieur. Dans la mesure où ce niveau est essentiellement financé par des fonds publics dans presque tous les pays européens, la plupart subissent des restrictions budgétaires importantes. En même temps, les politiques d'ouverture de l'enseignement supérieur se sont imposées sous la pression des exigences de qualification de plus en plus élevée et des besoins du monde de l'entreprise. La réduction des opportunités de trouver un emploi a accentué la demande de formation et le chômage a entraîné le retour des adultes les moins qualifiés à l'éducation. Les systèmes d'enseignement supérieur sont ainsi pris dans une double contradiction : accueillir en leur sein une masse de plus en plus importante de jeunes sans disposer des moyens pour faire face à cet afflux.

Diverses mesures sont prises un peu partout. Elles varient selon les différentes structures préexistantes, les modes d'accès et l'organisation en place. On peut cependant relever un certain nombre de tendances communes. Ainsi, l'autonomie dont, traditionnellement, jouissaient seules les institutions supérieures au Royaume-Uni s'est élargie aux systèmes d'enseignement supérieur de nombreux pays. Généralement justifiées dans le souci d'accroître la responsabilité des institutions en matière de qualité et de sensibilité aux besoins locaux, ces réformes sont à mettre en relation avec la réduction des financements publics. Comme le souligne G. Neave', les institutions sont ainsi devenues elles-mêmes 
responsables des choix à opérer dans l'utilisation de leurs budgets, y compris souvent l'éventail des cours offerts et le nombre d'étudiants admis. Ce processus de décentralisation est largement entamé au Portugal, en Espagne, au Danemark, aux PaysBas et en Finlande. En contraste à ce mouvement, quelques pays dont la France, l'Allemagne, la Grèce, l'Autriche ont conservé un contrôle central important. Ce processus d'autonomie se combine partout avec une supervision externe de contrôle de la qualité par l'administration centrale. Les exigences accrues de qualité et de justification du rapport coût/efficacité se font de plus en plus pressantes de la part des gouvernements. Dans certains pays, l'évaluation du rendement et de la qualité des enseignements est progressivement mise en place. Elle peut être réalisée sur une base interne d'autoévaluation comme aux Pays-Bas ou imposée par un organe extérieur comme au Danemark et au Royaume-Uni. Dans ces deux derniers pays, la dépendance du financement des institutions aux résultats de telles évaluations est progressivement mise en place. Ces nouveaux modes de financement liés aux résultats (output fundings) sont discutés dans d'autres pays.

Afin de répondre à la demande croissante de formation, l'organisation traditionnelle binaire de l'enseignement supérieur en secteurs universitaire et non universitaire s'estompe progressivement. Des institutions non universitaires délivrent aujourd'hui des diplômes de niveau universitaire. On citera par exemple, les Hochschulen en Allemagne et les Escuelas Tecnicas Supériores en Espagne. Cette tendance se marque aussi par l'accès possible au doctorat à partir de l'enseignement supérieur non universitaire comme en Communauté flamande de Belgique, en Grèce, en France et en Autriche. L'offre de formations professionnelles courtes, appartenant traditionnellement au niveau de l'enseignement secondaire, est en expansion dans le niveau supérieur. Dans plusieurs pays, elles sont mêmes intégrées au niveau universitaire. Dans d'autres, cette ouverture de l'enseignement supérieur à des formations qualifiantes professionnelles joue le rôle de maintien ou préservation du statut plus élevé des formations académiques traditionnelles.

Parallèlement, vu le nombre limité de places et les restrictions budgétaires, des stratégies sont adoptées pour essayer de maîtriser la demande croissante. Les problèmes sont particulièrement marqués dans les pays non sélectifs offrant un accès largement ouvert à l'enseignement supérieur. La limitation par la mise en place de systèmes numerus clausus s'implante progressivement au moins dans certaines facultés très demandées comme la médecine, la dentisterie et les études vétérinaires. Les abandons, les échecs en cours de formation et le prolongement excessif des études ont attiré de plus en plus l'attention des politiques. Ils font l'objet de mesures par exemple, en Belgique, en Espagne, en France, au Portugal, aux Pays-Bas où une supervision et des services de guidance sont mis en place pour conseiller et réorienter si nécessaire les étudiants dans leur choix. Plusieurs pays visent également à réduire la durée des études et les redoublements en limitant le temps supplémentaire dont un étudiant peut bénéficier en plus de la durée théorique prévue pour mener à bien ses études. C'est le cas par exemple en Belgique et aux Pays-Bas. Dans d'autres pays, la durée de certaines formations de premier niveau ont été réduites (Allemagne, Finlande, Danemark).

21 On peut s'en réjouir, depuis la période d'après guerre, les systèmes éducatifs se sont laissés pénétrer des nouvelles idées sans jamais remettre en cause les principes fondamentaux de la démocratisation de l'accès à l'éducation. Il faut espérer aujourd'hui que les exigences de qualité impulsées par les politiques néolibérales acceptent les limites 
de la mise en place d'un système compétitif et ne détruisent pas tous les acquis du passé. Alors, les sociétés européennes pourront réussir la « révolution éducative » tant attendue pour aborder efficacement le siècle prochain sans laisser sur le côté tous ceux qui n'auront pas pu suivre.

\section{NOTES}

1. Cet article porte uniquement sur les enjeux des systèmes éducatifs et exclut la formation professionnelle proprement dite.

2. Enseigner et apprendre, vers la société cognitive, Livre blanc, Commission européenne, 1995. Accomplir l'Europe par l'éducation et la formation, Rapport du groupe de réflexion, DGXXII, Commission européenne, 1996.

3. L'éducation, un trésor est caché dedans, Commission sur l'éducation au XXI ${ }^{\mathrm{e}}$ siècle, UNESCO, Éditions Odile Jacob, 1996.

4. Dix années de réformes au niveau de l'enseignement obligatoire dans l'Union européenne, Bruxelles, Eurydice, 1996.

5. Chiffres clés de l'éducation dans l'Union européenne, Commission européenne, Eurydice, 1996.

6. L'enseignement secondaire dans l'Union européenne, ses structures, son organisation pédagogique, son administration, Bruxelles, Eurydice, 1997.

D. Kallen, What Secondary Education for a Changing Europe? Trends, Challenges and Prospects, Rapport final du Conseil de l'Europe, décembre 1996.

7. « Key Competences in Europe », European Journal of Education, Vol. 32, n 1, 1997, p. 45-58.

8. Pour une information plus détaillée, lire: Les chefs d'établissements scolaires dans l'Union européenne et Conseils consultatifs et autres formes de participation sociale, Bruxelles, Eurydice, 1996.

9. G. Neave, "The Politics of Quality: Developments in Higher Education in Western Europe", European Journal of Education, vol. 29, n 2, 1994, p. 115-134.

\section{RÉSUMÉS}

La recherche d'un équilibre entre l'autonomie des acteurs de l'éducation et la garantie de qualité de l'enseignement constitue un des enjeux de l'évolution des systèmes éducatifs en Europe. Deux objectifs prioritaires sont assignés à l'éducation : l'égalité d'accès à des compétences de base, d'une part, et, d'autre part, la transformation de l'enseignement supérieur pour répondre aux demandes croissantes de formation. 
INDEX

Mots-clés : autonomie, enseignement supérieur, établissement d'enseignement, politique éducative, qualité de l'enseignement, système éducatif

Index géographique : Europe

\section{AUTEUR}

\section{ARLETTE DELHAXHE}

Unité européenne Eurydice, Bruxelles, Belgique. 